\title{
Mathematical Integration for Solving Biological Growth in Fish Lake Problem Using Gompertz Approach
}

\author{
Samuel Olukayode Ayinde ${ }^{*}$, Roseline Bosede Ogunrinde \\ Department of Mathematics, Faculty of Science, Ekiti State University, Ado Ekiti, Nigeria \\ Email address: \\ ayinde.olukayode@eksu.edu.ng (S. O. Ayinde), roseline.ogunrinde@eksu.edu.ng (R. B. Ogunrinde) \\ ${ }^{*}$ Corresponding author
}

To cite this article:

Ayinde Samuel Olukayode, Ogunrinde Roseline Bosede. Mathematical Integration for Solving Biological Growth in Fish Lake Problem Using Gompertz Approach. Biomedical Statistics and Informatics. Vol. 3, No. 3, 2018, pp. 43-48. doi: 10.11648/j.bsi.20180303.11

Received: July 6, 2018; Accepted: August 3, 2018; Published: August 31, 2018

\begin{abstract}
A lake is classified as a body of relatively still water that is almost completely surrounded by land with a river or stream that feeds into it or drains from it. A lake that has fish that you can catch can either be man-made or natural, with natural lakes tending to have more successful results. In this research, an interpolating function was proposed following Gompertz function approach considering the scale and shape parameters, a Numerical Method was developed and applied to solve the biological fish lake stocking and growth problem which gives effective results as when Gompertz equation was used directly. Numerical method is an effective tool to solve the problem of growth as its applicable in Gompertz equation. The method results obtained found to be favourable when the Numerical Solution and Analytical Solution is compared as the error obtained is minimal showing the effectiveness of the Method. Gompertz Function or equation was for long of interest only to actuaries and demographics. Its however, recently been used by various authors as a growth curve or function both for biological, economics and Management phenomena. Therefore, we have been able to show how the numerical integration obtained from the interpolating function work the same way Gompertz function worked.
\end{abstract}

Keywords: Gompertz Equation, Mathematical Integration, Logistic Growth, Carrying Capacity

\section{Introduction}

Freshwater Lakes are in very different places, so is the rushing oceans of the world. The creatures that live in them are different to one another in nature, among which is the fish. It is generally known that we have Lake (freshwater) fish and ocean (saltwater) fish. These lakes or oceans have diverse fish population and the growth of a lake or ocean fish depends on the nutrients, minerals and the food available.

A fishery is an area with an associated fish or aquatic population, which is harvested for its commercial or recreational value. Fisheries can be wild or farmed with various population dynamics. [1]

Population dynamics describe the ways in which a given population grows and shrinks over time, as controlled by birth, death and migration. It is the basis for understanding changing fishery patterns and issues such as habitat destruction, predation and optimal harvesting rates. [2]

As a result of acidification, reproduction of fish fails and population densities decrease. The growth of fish is most clearly affected at a late stage of acidification $(\mathrm{pH}<5)$, when the only fish remaining are those in the sparse populations of fish, then few fish have plenty of food, and they can grow remarkably fast. Despite their good tolerance to acidity, small fish may grow slowly. However, if they find something to displace fish food, they are likely to grow normally at least during their early years of life. Though certain kind of whitefish tolerates acidity. The growth of whitefish was faster in lakes without roach than in lakes that had thriving roach populations.

The population dynamics of fisheries are used by fisheries scientists to determine sustainable yields, $[3,4]$ The basic accounting relation for population dynamics is the BIDE (Birth, Immigration, Death, Emigration) model shown as

$$
N_{1}=N_{0}+B-D+I-E
$$

Where $N_{1}$ is the number of individuals at time $1, N_{0}$ is the number of individuals at time $0, B$ is the number of individuals born, $D$ is the number that died, $I$ is the number 
that immigrated, and $E$ is the number that emigrated between time 0 and time 1. [5]

Caswel (2001) [6] stated that a fishery population is affected by three dynamic rate functions: (i) Birth rate, (ii) Growth rate (iii) Mortality. He reiterated that harvestable surplus is the number of individuals that can be harvested from the population without affecting long term stability.

Stocking a fish lake involves (i) Minimum Viable Population, which is a lower bound on the population of a species, such that it can survive in the wild. That is the smallest possible size at which a biological population can exist without facing extinction from natural disasters or demographic, environmental or genetic factor. (ii) Maximum Sustainable Yield which in population ecology and economics, the maximum sustainable yield is, theoretically, the largest catch that can be taken from a fishery stock over an indefinite period [2]. Under the assumption of logistic growth, the maximum sustainable yield will be exactly at half the carrying capacity of a species, as this is the stage at which population growth is the highest. The maximum sustainable yield is usually higher than the optimum sustainable yield. [7]

Moreover, the logistic model of growth is produced by a population introduced to a new habitat or with very poor numbers going through a lag phase of slow growth at first. Once it reaches a foothold population, it will go through a rapid growth rate that will start to level off once the species approaches carrying capacity. [8]

The main objective of this paper was to propose an interpolating function using the Gompertz approach with two parameters defined, developed a Numerical Integration to evaluate the growing population of a biological stocked lake. In order to test the reliability and ability of the function cum integration, the experimental data, a MAT 274, HM 2 problem and solution proposed by Bin Cheng (2011) was considered. [9]

\section{Method}

\subsection{Interpolating Function}

Most phenomena that occur nowadays in the field of physical, chemical, biological and management sciences can be modeled in the form of different equations. The solutions to most differential equations of this type got solutions through the approximate solutions that are generated by means of numerical techniques via the formulation of Interpolating Function. [10]

Therefore, let us assume that the theoretical solution $y(x)$ to the initial value problem

$$
y^{\prime}=f(x, y), y\left(x_{0}\right)=y_{0}
$$

can be represented in the interval $\left[x_{n}, x_{n+1}\right], n \geq 0$ by the non-polynomial interpolating function;

$$
F(x, y)=K\left[\alpha_{1} e^{\beta x}+\alpha_{2} B^{x}+\alpha_{3} \cos x\right]
$$

Where $\alpha_{1}, \alpha_{2}, \alpha_{3}$ are real undetermined coefficients, $\beta$ and $B$ are the shapes and scale parameters, $\mathrm{K}$ represent the saturation level using Gompertz approach. The intervals defined are $x \in[0,1]$ and $k \in(0,1]$. Gompertz equation or function is a sigmoid function developed by the Jewish Mathematician, Benjamin Gompertz in 1938 as it was used to described the growth in cells of animate or in-animates. The important conclusion of Gompertz was that the force of mortality (practically the same as the rate of mortality) increased in geometric progression with age. The actual Gompertz equation as proposed by [1] is

$$
y=K e^{-e^{a-b x}},
$$

where $x=\frac{a}{b}$ is the point of inflection, $a$ and $b$ in compared with $\beta$ and $B$ in (3) are the same.

We shall assume $y_{n}$ is a numerical estimate to the theoretical solution $y(x)$ and $f_{n}=f\left(x_{n}, y_{n}\right)$, and define mesh points as follows:

$$
x_{n}=a+n h, x_{n}=a+(n+1) h, n=0,1,2, \ldots
$$

We can impose the following constraints on the interpolating function (3) in order to get the undetermined coefficients. The interpolating function must coincide with the theoretical solution at $x=x_{n}$ and $x=x_{n+1}$. Hence it is required that (3) can be written as

$$
F\left(x_{n}, y_{n}\right)=K\left(\alpha_{1} e^{\beta x_{n}}+\alpha_{2} B^{x_{n}}+\alpha_{3} \cos x_{n}\right)
$$

and

$F\left(x_{n+1}, y_{n+1}\right)=K\left(\alpha_{1} e^{\beta x_{n+1}}+\alpha_{2} B^{x_{n+1}}+\alpha_{3} \cos x_{n+1}\right)$

\subsection{Derivation of the Numerical Integration}

The derivatives of the interpolating function (3) are required to coincide with the differential equation as well as its first, second, and third derivatives with respect to $x$ at $x=x_{n}$

We denote the $i$-th total derivatives of $f(x, y)$ with respect to $x$ with $f^{(i)}$ such that

$$
F^{1}\left(x_{n}\right)=f_{n}, F^{2}\left(x_{n}\right)=f_{n}^{1}, F^{3}\left(x_{n}\right)=f_{n}^{2}
$$

This implies that,

$$
\begin{gathered}
f_{n}=k \alpha_{1} \beta e^{\beta x_{n}}+k \alpha_{2} B^{x_{n}} \log B-k \alpha_{3} \sin x_{n} \\
f_{n}{ }^{1}=k \alpha_{1} \beta^{2} e^{\beta x_{n}}+k \alpha_{2} B^{x_{n}}(\log B)^{2}-k \alpha_{3} \cos x_{n} \\
f_{n}{ }^{2}=k \alpha_{1} \beta^{3} e^{\beta x_{n}}+k \alpha_{2} B^{x_{n}}(\log B)^{3}+k \alpha_{3} \sin x_{n}
\end{gathered}
$$

\subsection{Forming System of Equation}

To obtain values for $\alpha_{1}, \alpha_{2}$, and $\alpha_{3}$ from the system (9) to (11), we have 


$$
\left(\begin{array}{ccc}
K \beta e^{\beta x_{n}} & K B^{x_{n}} \log B & -K \sin x_{n} \\
K \beta^{2} e^{\beta x_{n}} & K B^{x_{n}}(\log B)^{2} & -K \cos x_{n} \\
K \beta^{3} e^{\beta x_{n}} & K B^{x_{n}}(\log B)^{3} & K \sin x_{n}
\end{array}\right)\left(\begin{array}{c}
\alpha_{1} \\
\alpha_{2} \\
\alpha_{3}
\end{array}\right)=\left(\begin{array}{c}
f_{n} \\
f_{n}{ }^{1} \\
f_{n}{ }^{2}
\end{array}\right)
$$

Taking this as a system of equations, $A X=B$, it gives

$$
\begin{aligned}
& f_{n}(\log B)^{2} \sin x_{n}+(\log B)^{3} \cos x_{n}-(\log B)\left(f_{n}^{1} \sin x_{n}+f_{n}^{2} \cos x_{n}\right) \\
& \alpha_{1}=\frac{-\sin x_{n}\left(f_{n}^{1}(\log B)^{3}-f_{n}^{2}(\log B)^{2}\right)}{K \beta e^{\beta x_{n}}\left((\log B)^{2} \sin x_{n}+(\log B)^{3} \cos x_{n}\right)-K \log B e^{\beta x_{n}}\left(\beta^{2} \sin x_{n}+\beta^{3} \cos x_{n}\right)} \\
& \left.-K \sin x_{n} e^{\beta x_{n}}\left(\beta^{2}(\log B)^{3}-\beta^{3}(\log B)^{2}\right)\right) \\
& \alpha_{2}=\frac{\beta\left(f_{n}^{1} \sin x_{n}+f_{n}^{2} \cos x_{n}\right)-f_{n}\left(\beta^{2} \sin x_{n}+\beta^{3} \cos x_{n}\right)-\sin x_{n}\left(\beta^{2} f_{n}^{2}-\beta^{3} f_{n}^{1}\right)}{K \beta B^{x} n\left((\log B)^{2} \sin x_{n}+(\log B)^{3} \cos x_{n}\right)-K B^{x} n \log B\left(\beta^{2} \sin x_{n}+\beta^{3} \cos x_{n}\right)} \\
& \left.-K B^{x_{n}} \sin x_{n}\left(\beta^{2}(\log B)^{3}-\beta^{3}(\log B)^{2}\right)\right) \\
& \alpha_{3}=\frac{\beta\left(\log B^{2} f_{n}^{2}-\log B^{3} f_{n}^{1}\right)-\log B\left(\beta^{2} f_{n}^{2}-\beta^{3} f_{n}^{1}\right)+f_{n}\left(\beta^{2} \log B^{3}-\beta^{3} \log B^{2}\right)}{K \beta B^{x} n\left((\log B)^{2} \sin x_{n}+(\log B)^{3} \cos x_{n}\right)-K B^{x} \log B\left(\beta^{2} \sin x_{n}+\beta^{3} \cos x_{n}\right)} \\
& \left.-K B^{x} n \sin x_{n}\left(\beta^{2}(\log B)^{3}-\beta^{3}(\log B)^{2}\right)\right)
\end{aligned}
$$

Since

$$
F\left(x_{n+1}\right)=y\left(x_{n+1}\right) \text { and } F\left(x_{n}\right)=y\left(x_{n}\right)
$$

This implies that

$$
\begin{aligned}
& y\left(x_{n+1}\right)=y_{n+1} \text { and } y\left(x_{n}\right)=y_{n} \\
& F\left(x_{n+1}\right)-F\left(x_{n}\right)=y_{n+1}-y_{n}
\end{aligned}
$$

Therefore we have from (6), (7) into (16)

$$
\begin{gathered}
y_{n+1}-y_{n}=K\left(\alpha_{1} e^{\beta x_{n+1}}+\alpha_{2} B^{x_{n+1}}+\alpha_{3} \cos x_{n+1}\right)+K\left(\alpha_{1} e^{\beta x_{n}}+\alpha_{2} B^{x_{n}}+\alpha_{3} \cos x_{n}\right) \\
=K \alpha_{1}\left[e^{\beta x_{n+1}}-e^{\beta x_{n}}\right]+K \alpha_{2}\left[B^{x_{n+1}}-B^{x_{n}}\right]+K \alpha_{3}\left[\cos x_{n+1}-\cos x_{n}\right]
\end{gathered}
$$

Recall that

$x_{n}=a+n h, x_{n+1}=a+(n+1) h \quad$ with $\quad n=$

$0,1,2 \ldots$ Therefore, by expansion of (18)

$$
\begin{gathered}
y_{n+1}-y_{n}=K \alpha_{1} e^{\beta x_{n}}\left(e^{\beta h}-1\right)+K \alpha_{2} B^{x_{n}}\left(B^{h}-1\right)+ \\
K \alpha_{3}\left[\cos \left(x_{n}+h\right)-\cos x_{n}\right]
\end{gathered}
$$

\subsection{The Numerical Integration}

Equation (19) can be written as

$$
\begin{gathered}
y_{n+1}=y_{n}+P+Q+R \\
P=\frac{\left(e^{\beta h}-1\right)\left[\left(f_{n}(\log B)^{2}-f_{n}^{1} \log B-f_{n}^{1}(\log B)^{3}+f_{n}^{2}(\log B)^{2}\right) \sin x_{n}+\left(f_{n}(\log B)^{3}-f_{n}^{2} \log B\right) \cos x_{n}\right]}{\left(\beta(\log B)^{2}-\beta^{2} \log B-\beta^{2}(\log B)^{3}+\beta^{3}(\log B)^{2}\right) \sin x_{n}+\left(\beta(\log B)^{3}-\beta^{3} \log B\right) \cos x_{n}} \\
Q=\frac{\left(B^{h}-1\right)\left[\left(\beta f_{n}^{1}-\beta^{2} f_{n}-\beta^{2} f_{n}^{2}+\beta^{3} f_{n}^{1}\right) \sin x_{n}+\left(\beta f_{n}^{2}-\beta^{3} f_{n}\right) \cos x_{n}\right]}{\left(\beta(\log B)^{2}-\beta^{2} \log B-\beta^{2}(\log B)^{3}+\beta^{3}(\log B)^{2}\right) \sin x_{n}+\left(\beta(\log B)^{3}-\beta^{3} \log B\right) \cos x_{n}} \\
R=\frac{\left[\cos \left(x_{n}+h\right)-\cos x_{n}\right]\left[\beta f_{n}^{2} \log B^{2}-\beta^{2} f_{n}^{2} \log B-\beta^{2} f_{n} \log B^{3}-\beta f_{n}^{1} \log B^{3}+\beta^{3} f_{n}^{1} \log B+\beta^{3} f_{n} \log B^{2}\right]}{\left(\beta(\log B)^{2}-\beta^{2} \log B-\beta^{2}(\log B)^{3}+\beta^{3}(\log B)^{2}\right) \sin x_{n}+\left(\beta(\log B)^{3}-\beta^{3} \log B\right) \cos x_{n}}
\end{gathered}
$$

Equation (21) is the new numerical integration for solution of the first order differential equation. This numerical integration has been tested using test equation and on some initial value problems of first order differential equations. [11]

\section{Experimental Data}

Fish Population Problem (1). [9]

Biologist stocked a lake with 500 fish and estimated the carrying capacity (the maximal population for the fish of that species in that lake) to be 5300 . The number of fish doubled 
in the first year.

(a) Assuming that the size of the fish population satisfied the logistic equation

$$
\frac{d P}{d t}=k P\left(1-\frac{P}{K}\right)
$$

Solve the equation to find an expression for the size of the population after $t$ years.

(b) How long will it take for the population to increase to 2650 .

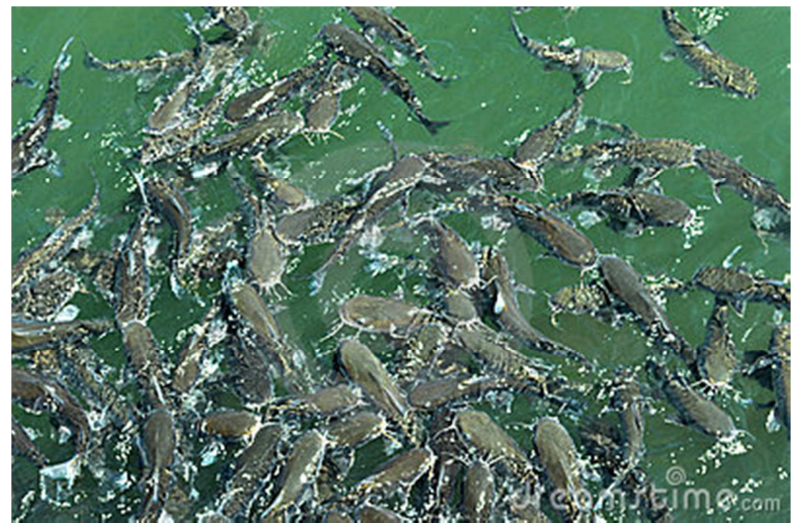

Source: fish-feeding 2017(dicomstime.com)

Figure 1. Fish Population during feeding.

\section{SOLUTION}

Assuming that the size of the fish population satisfies the logistic equation $\frac{d P}{d t}=k P\left(1-\frac{P}{K}\right)$, If the carrying capacity is given as $K=5300$,

Using separable differential equation, $\frac{d P}{d t}=\frac{k}{5300} P(5300-$ P)

Solve by partial fraction, integrate and apply exponential, we have

$$
\frac{P}{5300-P}=C_{1} e^{k t}
$$

At $P(0)=500$ and set $t=0, P=500$ in (23) we have $C_{1}=0.104166666 \ldots$

Hence

(a) If carrying capacity is given as $\mathrm{K}=5300,500$ fish at the beginning and fish doubled after a year.

i.e. $P(0)=500, P(1)=1000$. When $(23)$ is solved, the analytical solution is

$$
\begin{gathered}
\mathrm{P}(\mathrm{t})=26500 /(5+48 * \exp (-\mathrm{k} * \mathrm{t})), P(0)=500(24) \\
\mathrm{P}(\mathrm{t})=53000 * \exp (-\mathrm{k}) /(10 * \exp (-\mathrm{k})+43 * \exp (-\mathrm{k} * \\
\mathrm{t})), P(1)=1000
\end{gathered}
$$

Then we can solve the problem by using the numerical integration (21), hence

\section{Results}

Table 1. Result of Solution to problem 1(a), size of fish population in the interval $0 \leq x \leq 1, h=0.1, B=2.3211, \beta=182.33845$.

\begin{tabular}{llll}
\hline Xn & Numerical & Exact & Absolute \\
\hline & Solution & Solution & Error \\
\hline 0.00 & 500.0000000000 & 500.0000000000 & $0.0000 \mathrm{E}-6$ \\
0.10 & 537.5503454020 & 537.5503473278 & $1.9258 \mathrm{E}-6$ \\
0.20 & 577.6081405140 & 577.6081385840 & $1.9300 \mathrm{E}-6$ \\
0.30 & 620.2622238788 & 620.2622258146 & $1.9356 \mathrm{E}-6$ \\
0.40 & 665.6221812757 & 665.6221793329 & $1.9428 \mathrm{E}-6$ \\
0.50 & 713.7933557078 & 713.7933537560 & $1.9518 \mathrm{E}-6$ \\
0.60 & 764.8753168267 & 764.8753148647 & $1.9620 \mathrm{E}-6$ \\
0.70 & 818.9601238500 & 818.9601218746 & $1.9754 \mathrm{E}-6$ \\
0.80 & 876.1304796367 & 876.1304776457 & $1.9910 \mathrm{E}-6$ \\
0.90 & 936.4577865282 & 936.4577657082 & $2.08200 \mathrm{E}-5$ \\
1.00 & 1000.0000201282 & 1000.0000000000 & $2.01282 \mathrm{E}-5$ \\
\hline
\end{tabular}

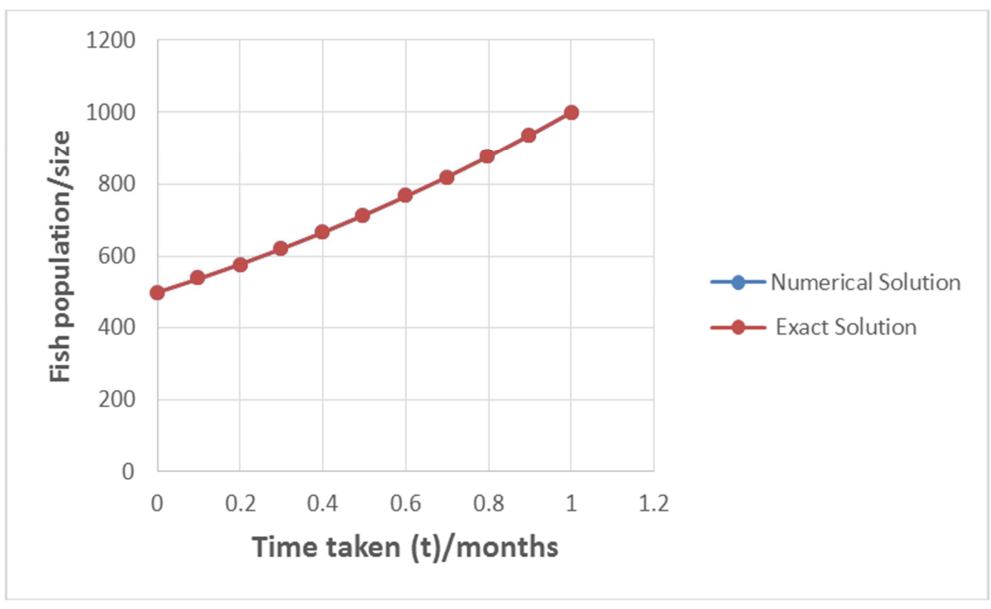

Figure 2. The graph of the Numerical and Exact solution of the increase in size of the fish population after tyears. 
Comparing the Numerical Solution to the exact solution, it is seen that the absolute error is minimal, which showed the effectiveness of the method. However, when compared with manual computation it also performed better.

(b) The time $t$ it takes for the population to increase to 2650 .

Table 2. Result of Solution to problem $1 b$, population increase to 2650 Using Integration (21) to solve the initial value problem (25) in the interval $0 \leq x \leq$ $12, h=0.1, B=2.3211, \beta=182.3392$.

\begin{tabular}{|c|c|c|c|}
\hline \multirow{2}{*}{$\mathbf{X n}$} & Numerical & Exact & Absolute \\
\hline & Solution & Solution & Error \\
\hline 0.00 & 500.0000000000 & 500.0000000000 & 0.0000000000 \\
\hline 0.10 & 537.5730163704 & 537.5729207903 & 0.0000955801 \\
\hline 0.20 & 577.6295000000 & 577.6295177324 & 0.0001000002 \\
\hline 0.30 & 620.2822325019 & 620.2821325009 & 0.0001000010 \\
\hline 0.40 & 665.6404124983 & 665.6403124963 & 0.0001000020 \\
\hline 0.50 & 713.8094898412 & 713.8093898384 & 0.0001000028 \\
\hline 0.60 & 764.8890086761 & 764.8889086721 & 0.0001000040 \\
\hline 0.70 & 818.9710079491 & 818.9709079436 & 0.0001000055 \\
\hline 0.80 & 876.1381721782 & 876.1380721711 & 0.0001000071 \\
\hline 0.90 & 936.4618690903 & 936.4617690820 & 0.0001000083 \\
\hline 1.00 & 1000.0001000101 & 1000.0000000000 & 0.0001000101 \\
\hline 1.10 & 1066.7953963664 & 1066.7952963556 & 0.0001000108 \\
\hline 1.20 & 1136.8727034052 & 1136.8726033932 & 0.0001000120 \\
\hline 1.30 & 1210.2372997351 & 1210.2371997218 & 0.0001000133 \\
\hline 1.40 & 1286.8728083823 & 1286.8727083676 & 0.0001000147 \\
\hline 1.50 & 1366.7393609820 & 1366.7392609658 & 0.0001000162 \\
\hline 1.60 & 1449.7719811368 & 1449.7718811180 & 0.0001000188 \\
\hline 1.70 & 1535.8792552405 & 1535.8791552165 & 0.0001000240 \\
\hline 1.80 & 1624.9423586893 & 1624.9422586618 & 0.0001000275 \\
\hline 1.90 & 1716.8145019731 & 1716.8144019403 & 0.0001000328 \\
\hline 2.00 & 1811.3208542131 & 1811.3207541643 & 0.0001000488 \\
\hline 2.10 & 1908.2589913410 & 1908.2588912847 & 0.0001000563 \\
\hline 2.20 & 2007.3999024050 & 2007.3998023460 & 0.0001000590 \\
\hline 2.30 & 2108.4895702941 & 2108.4894702305 & 0.0001000636 \\
\hline 2.40 & 2211.2511240067 & 2211.2510239384 & 0.0001000683 \\
\hline 2.50 & 2315.3875385312 & 2315.3874384562 & 0.0001000750 \\
\hline 2.60 & 2420.5848368301 & 2420.5847367499 & 0.0001000802 \\
\hline 2.70 & 2526.5157276970 & 2526.5156276106 & 0.0001000864 \\
\hline 2.80 & 2632.8435943385 & 2632.8434942483 & 0.0001000902 \\
\hline 2.90 & 2739.2267329846 & 2739.2266328874 & 0.0001000972 \\
\hline 3.00 & 2845.3227293218 & 2845.3226292198 & 0.0001001020 \\
\hline
\end{tabular}

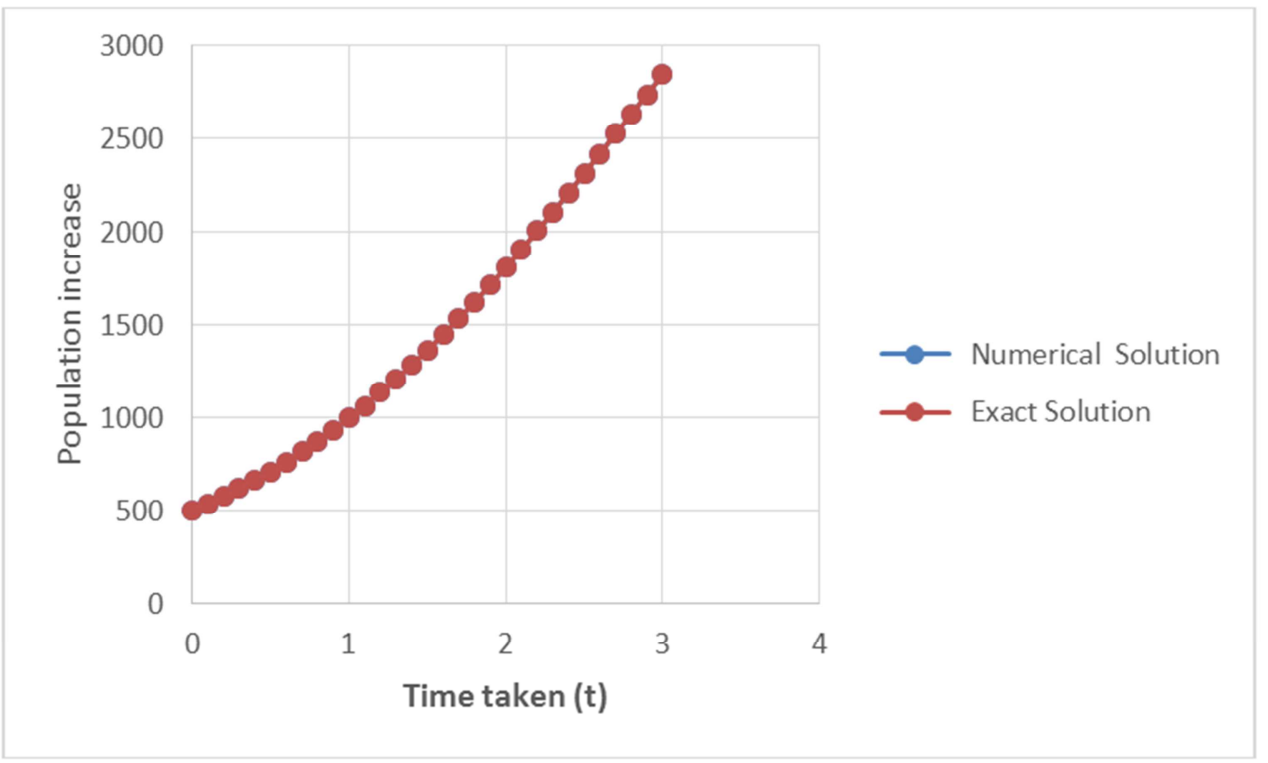

Figure 3. The graph of the Numerical and Exact solution at the time (t) takes for the population to increase to 2650.

At this end, the exact value of the population increase to 2650 fish between the range $t=2.80$ and $t=2.90$ which when calculated averagely, the time $(\mathrm{t})$ is 2.82 (approx.). 


\section{Discussion}

The problem here was solved numerically with the formula (21) and the results obtained are shown in table 1 and 2 . In Table 1, the results showed the size of fish population increase from 500 to 1000 . It can be seen that the discretization errors obtained from the solutions are reasonable and is of the low order. Table 2 moreover, showed the time it takes for the population of the fish to increase to 2650. From the results, as the Gompertz equation was a very realistic model or equation to measure growth, so also the method (21) is reliable derived from the Interpolating function (3).

\section{Conclusion}

The solution to this problem is not exhaustive at this point. The basis of this research is to show how numerical integration can be used to solve such a problem of this type. Many authors that solved this problem either solve it analytically or using direct method as it was used by [9] Gompertz equation where the two parameters are considered will be a good tool for solving biological problems numerically, though this is an approximate solution.

\section{References}

[1] Winsor, C. P. (1932). "The Gompertz Curve as a Growth Curve". Proceedings of the National Academy of Sciences. Vol 18. No 1, 1-7.

[2] Wilderbuera, T. K. and Zhang, C. (1999). "Evaluation of the Population Dynamics and Yield". Characterisitics of Alaska place, Pleuronects quadrituberculatus in the Easten Bering Sea. Fisheries Research. Vol. 41, issue 2.
[3] Richard W. Z., Chris J. H., Stephen L. K., Thomas P. G., and Philip S. L. (2003). "Ecologically Sustainable Yield". Archived 2011/06/11 at the Wayback Machine. American Scientist. March-April.

[4] Mickens, R. E. (1994). "Non-Standard Finite Difference Models of Differential Equations". World Scientific, Singapore.

[5] Caswell, H. (2001). "Matrix Population Models: Construction, Analysis and Interpretation". $2^{\text {nd }}$ Edition, Sinauer Associates. Sunderland, Maassachusetts.

[6] Ogunrinde R. B., and Ayinde S. O. (2017). "A Numerical Integration for Solving First Order Differential Equation Using Gompertz Function Approach". American Journal of Computational and Applied Mathematics. 7(6): 143 - 148.

[7] Caswell, H. (2006). "Population Models: Analysis and Interpretation". $3^{\text {rd }}$ Edition, Sinauer Associates. Sunderland, Maassachusetts.

[8] Walters C. and Maguire J. (1996). "Lessons for Stock assessment from the Northern Cod Collapse". Reviews in fish Biology and Fisheries. 6:125-137

[9] Richards F. J. (1959). "A Flexible Growth Function for Empirical Use". Journal of Experimental Bothany. 10:290301 .

[10] Cheng B. (2011). "MAT274 HM 2 Solutions". Arisona State University. School of Mathematical and Statistical Sciences. USA.

[11] www://en.m.wikipedia.org/.../Population 2017/03/12.

[12] Kapur V., Troy D. and Oris J. (1997). "A Sustainable Fishing Simulation Using Mathematical Modeling." Crossroads. 